\title{
A local limit theorem for directed polymers in random media: the continuous and the discrete case
}

\author{
Vincent Vargas ${ }^{1}$ \\ Université Paris 7, Mathématiques, case 7012, 2, place Jussieu, 75251 Paris, France \\ Received 1 March 2005; accepted 30 August 2005 \\ Available online 19 January 2006
}

\begin{abstract}
In this article, we consider two models of directed polymers in random environment: a discrete model in a general random environment and a continuous model. We consider these models in dimension greater than or equal to 3 and we suppose that the normalized partition function is bounded in $L^{2}$ (the "high" temperature case). Under these assumptions, Sinai proved in [Y. Sinai, A remark concerning random walks with random potentials, Fund. Math. 147 (1995) 173-180] a local limit theorem for the discrete model, using a perturbation expansion. In this article, we give a new method for proving Sinai's local limit theorem. This new method can be transposed to the continuous setting in which we prove a similar local limit theorem.
\end{abstract}

(c) 2005 Elsevier SAS. All rights reserved.

\section{Résumé}

Dans cet article, on considère deux modèles de polymères dirigés en environnement aléatoire : un modèle discret en environnement aléatoire général et un modèle continu. On considère ces modèles en dimension supérieur ou égale à 3 et on suppose que la fonction de partition renormalisée est bornée dans $L^{2}$ (cela correspond au cas de «haute» température). Sous ces hypothèses, Sinai a montré dans [Y. Sinai, A remark concerning random walks with random potentials, Fund. Math., 147 (1995) 173-180] un théorème limite locale pour le modèle discret en utilisant un développement en perturbation. Dans cet article, on donne une nouvelle méthode pour démontrer le théorème limite locale ci-dessus. Cette nouvelle méthode peut être transposée au cas continu dans lequel on montre un théorème limite locale similaire.

(c) 2005 Elsevier SAS. All rights reserved.

MSC: 60K37; 60F05; 82B44; 82D60

Keywords: Directed polymers in random environment; Local limit theorem

\section{Introduction}

Directed polymers in random environment is a model of statistical mechanics in which stochastic processes interact with a random environment, depending on both time and space: one studies the path of the stochastic process under

E-mail address: vargas@math.jussieu.fr (V. Vargas).

1 Partially supported by CNRS (UMR 7599 "Probabilités et Modèles Aléatoires"). 
a random Gibbs measure depending on the temperature (as the temperature increases, the influence of the random environment decreases).

In this article, we will consider two polymer models: a random walk model of directed polymers and its continuous analogue, a Brownian model of directed polymers. The discrete model first appeared in the physics literature [6] to modelize the phase boundary of Ising model subject to random impurities and its first mathematical study was undertaken by Imbrie, Spencer in 1988 [7] and Bolthausen in 1989 [1]. The continuous model we study here was first introduced and studied by Comets and Yoshida in 2004 [3]. These models are related to many models of statistical physics. We refer to the survey paper [8] by Krug and Spohn for an account on these models and their relations.

In the sequel, we will suppose that the dimension of the underlying stochastic process is greater than or equal to 3 and that the normalized partition function is bounded in $L^{2}$ (see Sections 1.1-1.2 for the definition of the normalized partition function). Under these assumptions, the polymer is diffusive in the sense that a central limit theorem holds: by scaling by the square root of the length, the discrete and continuous polymer models converge in law to a Gaussian measure (see [7,1,11,5]). One can sometimes go a step further than convergence in law by giving an equivalent of the density: this is called a local limit theorem. In [10], Sinai obtained a local limit theorem by using a perturbation expansion. Unfortunately, it is not clear how to adapt the strategy to the continuous setting. The object of this work is to give a new method for proving Sinai's theorem; this method is sufficiently general to be easily adapted to prove a similar local limit theorem in the continuous setting. Our approach is simple and relies only on $L^{2}$ computations and on properties of the simple random walk bridges (Brownian bridges in the continuous case).

Finally, we recall that some results have been achieved in the case of dimension less than or equal to 2 or when the temperature is low. In these cases, the polymer is non-diffusive (see Remark 2.6 below) and many conjectures remain open. For an account on these cases, we refer to [2] in a Gaussian environment and to [4] in a general environment.

The article is organized as follows: each chapter is divided into two subchapters, one of them being devoted to the discrete model and the other one being devoted to the continuous model. First, we introduce the two models. In the second chapter, we will remind the known results at high temperature when the dimension of the underlying process is greater than or equal to 3; we will also formulate an analogue to Sinai's local limit theorem for the Brownian directed polymer. In the third chapter, we will prove the local limit theorem for both models.

\subsection{The random walk model of directed polymers}

- Let $\left(\left(\omega_{n}\right)_{n \in \mathbb{N}},\left(P^{x}\right)_{x \in \mathbb{Z}^{d}}\right)$ denote the simple random walk on the $d$-dimensional integer lattice $\mathbb{Z}^{d}$, defined on a measurable space $(\Omega, \mathcal{F})$; more precisely, for $x$ in $\mathbb{Z}^{d}$, under the measure $P^{x},\left(\omega_{n}-\omega_{n-1}\right)_{n \geqslant 1}$ are independent and

$$
P^{x}\left(\omega_{0}=x\right)=1, \quad P^{x}\left(\omega_{n}-\omega_{n-1}= \pm \delta_{j}\right)=\frac{1}{2 d}, \quad j=1, \ldots, d,
$$

where $\left(\delta_{j}\right)_{1 \leqslant j \leqslant d}$ is the $j$ th vector of the canonical basis of $\mathbb{Z}^{d}$. In the sequel, $P$ will denote $P^{0}$ and $P_{n}^{x}$ will denote the simple random walk measure on paths of length $n$ starting from $x$. For $x$ in $\mathbb{Z}^{d}$, let $q^{(n)}(x)$ be the probability for the random walk starting from 0 to be in $x$ at time $n$ :

$$
q^{(n)}(x) \stackrel{\text { def. }}{=} P\left(\omega_{n}=x\right) .
$$

- The random environment on each lattice site is a sequence $\eta=(\eta(n, x))_{(n, x) \in \mathbb{N} \times \mathbb{Z}^{d}}$ of real valued, non-constant and i.i.d. random variables defined on a probability space $(H, \mathcal{G}, Q)$ such that

$$
\forall \beta \in \mathbb{R} \quad \lambda(\beta) \stackrel{\text { def. }}{=} \ln Q\left(\mathrm{e}^{\beta \eta(n, x)}\right)<\infty .
$$

- For any $n>0$, we define the ( $Q$-random) polymer measure $\mu_{n}^{x}$ on paths of length $n$ starting from $x$ by:

$$
\mu_{n}^{x}(\mathrm{~d} \omega)=\frac{1}{Z_{n}^{x}} \exp \left(\beta H_{n}(\omega)-n \lambda(\beta)\right) P_{n}^{x}(\mathrm{~d} \omega)
$$

where $\beta \in \mathbb{R}$ is the inverse temperature,

$$
H_{n}(\omega) \stackrel{\text { def. }}{=} \sum_{j=1}^{n} \eta\left(j, \omega_{j}\right)
$$


and

$$
Z_{n}^{x}=P^{x}\left(\exp \left(\beta H_{n}(\omega)-n \lambda(\beta)\right)\right)
$$

is the normalized partition function $\left(Q\left(Z_{n}^{x}\right)=1\right)$.

Let $\left(\mathcal{G}_{n}\right)_{n \geqslant 0}$ be the filtration defined by

$$
\mathcal{G}_{n}=\sigma\left\{\eta(j, x) ; j \leqslant n, x \in \mathbb{Z}^{d}\right\} .
$$

For any fixed path $\omega,\left(\left(\sum_{j=1}^{n} \beta \eta\left(j, \omega_{j}\right)\right)-n \lambda(\beta)\right)_{n \geqslant 1}$ is a random walk with independent increments thus it is not hard to see that $\left(Z_{n}^{x}, \mathcal{G}_{n}\right)_{n \geqslant 0}$ is a positive martingale. Therefore, it converges $Q$-a.s. to a limit $Z_{\infty}^{x}$. Since the event $\left(Z_{\infty}^{x}=0\right)$ is measurable with respect to the tail $\sigma$-field

$$
\bigcap_{n \geqslant 1} \sigma\left\{\eta(j, x) ; j \geqslant n, x \in \mathbb{Z}^{d}\right\}
$$

by Kolmogorov's 0-1 law, there are only two possible situations

$$
Q\left(Z_{\infty}^{x}=0\right)=1 \quad \text { or } \quad Q\left(Z_{\infty}^{x}=0\right)=0 .
$$

In the former case, we say that strong disorder holds and in the latter case we say that weak disorder holds.

\subsection{The Brownian model of directed polymers}

- Let $\left(\left(\omega_{t}\right)_{t \in \mathbb{R}_{+}},\left(P^{x}\right)_{x \in \mathbb{R}^{d}}\right)$ denote a $d$-dimensional standard Brownian motion, defined on a measurable space $(\Omega, \mathcal{F})$. In the sequel, $P$ will denote $P^{0}$ and $P_{t}^{x}$ the Brownian measure on paths of length $t$ starting from $x$. For $t>0$ and $x, y$ in $\mathbb{R}^{d}$, let $p(t, x, y)$ be the transition density of the Brownian motion:

$$
p(t, x, y) \stackrel{\text { def. }}{=} \frac{1}{(2 \pi t)^{d / 2}} \mathrm{e}^{-|y-x|^{2} /(2 t)} .
$$

- The random environment $\eta$ is a Poisson point process on $\mathbb{R}_{+} \times \mathbb{R}^{d}$ with unit intensity, defined on a probability space $(M, \mathcal{G}, Q)$. We recall that $\eta$ is an integer valued random measure characterized by the following property: If $A_{1}, \ldots, A_{n} \in \mathcal{B}\left(\mathbb{R}_{+} \times \mathbb{R}^{d}\right)$ are disjoint and bounded Borel sets, then

$$
Q\left(\bigcap_{j=1}^{n}\left(\eta\left(A_{j}\right)=k_{j}\right)\right)=\prod_{j=1}^{n} \mathrm{e}^{-\left|A_{j}\right|} \frac{\left|A_{j}\right|^{k_{j}}}{k_{j} !}
$$

where $k_{1}, \ldots, k_{n} \in \mathbb{N}$ and |.| denotes the Lebesgue measure in $\mathbb{R}^{d+1}$. We define $V_{t}$ to be the unit volume "tube" around the graph $\left\{\left(s, \omega_{s}\right)\right\}_{0<s \leqslant t}$ of the Brownian path:

$$
\left.\left.V_{t}=V_{t}(\omega)=\{(s, x) ; s \in] 0, t\right], x \in U\left(\omega_{s}\right)\right\}
$$

where $U(x)$ is the closed ball in $\mathbb{R}^{d}$ with unit volume and centered at $x \in \mathbb{R}^{d}$.

- For any $t>0$, we define the ( $Q$-random) polymer measure $\mu_{t}^{x}$ on paths of length $t$ starting from $x$ by:

$$
\mu_{t}^{x}(\mathrm{~d} \omega)=\frac{\exp \left(\beta \eta\left(V_{t}\right)-\lambda(\beta) t\right)}{Z_{t}^{x}} P_{t}^{x}(\mathrm{~d} \omega),
$$

where $\beta \in \mathbb{R}$ is the inverse temperature and

$$
Z_{t}^{x}=P^{x}\left(\exp \left(\beta \eta\left(V_{t}\right)-\lambda(\beta) t\right)\right)
$$

is the normalized partition function $\left(Q\left(Z_{t}^{x}\right)=1\right)$. In this setting, the random environment is a Poisson point process so we get the explicit value:

$$
\left.\lambda(\beta)=\mathrm{e}^{\beta}-1 \in\right]-1, \infty[.
$$


It is natural to introduce the filtration $\left(\mathcal{G}_{t}\right)_{t>0}$ defined by :

$$
\left.\left.\mathcal{G}_{t}=\sigma\{\eta(A) ; A \in \mathcal{B}(] 0, t] \times \mathbb{R}^{d}\right)\right\} .
$$

As in the discrete setting, it is not hard to show that $\left(Z_{t}^{x}, \mathcal{G}_{t}\right)_{t>0}$ is a positive martingale which converges $Q$-a.s. to a non-negative random variable $Z_{\infty}^{x}$ that has the following property:

$$
Q\left(Z_{\infty}^{x}=0\right)=1 \text { or } Q\left(Z_{\infty}^{x}=0\right)=0 .
$$

In the former case, we say that strong disorder holds and in the latter case we say that weak disorder holds.

\section{Study of the directed polymers when the normalized partition function is bounded in $L^{2}(Q)$}

From now on, in the rest of this paper, we will only consider the case $d \geqslant 3$ and we will suppose that the normalized partition function is bounded in $L^{2}(Q)$. In that case, the latter converges $Q$-a.s. and in $L^{2}(Q)$ to the random variable $Z_{\infty}^{x}$. The $L^{2}$-convergence implies that $Q\left(Z_{\infty}^{x}\right)=1$ and therefore weak disorder holds. Under these assumptions, the behavior of the typical path under the polymer measure is diffusive (see [4] for the discrete case and [5] for the continuous case).

\subsection{The random walk model}

In order to get a nice probabilistic interpretation, we work on the product space $\left(\Omega^{2}, \mathcal{F}^{\otimes 2},\left(P^{x} \otimes P^{y}\right)_{x, y \in \mathbb{Z}^{d}}\right)$ and thus consider another simple random walk $\left(\widetilde{\omega}_{n}\right)_{n \in \mathbb{N}}$ independent of the first one $\left(\omega_{n}\right)_{n \in \mathbb{N}}$ under the same environment.

Let $\lambda_{2}(\beta) \stackrel{\text { def. }}{=} \lambda(2 \beta)-2 \lambda(\beta)$ and $N_{k, n}=N_{k, n}(\omega, \widetilde{\omega})$ be the number of ordered intersections of $\omega$ and $\widetilde{\omega}$ between $k$ and $n$ :

$$
N_{k, n} \stackrel{\text { def. }}{=} \sum_{j=k}^{n} 1_{\omega_{j}=\widetilde{\omega}_{j}} .
$$

With these notations, the following proposition is straightforward (e.g., [4]):

Proposition 2.1. We have the following identity:

$$
Q\left(\left(Z_{n}^{x}\right)^{2}\right)=P^{x} \otimes P^{x}\left(\mathrm{e}^{\lambda_{2}(\beta) N_{1, n}}\right)=P \otimes P\left(\mathrm{e}^{\lambda_{2}(\beta) N_{1, n}}\right) .
$$

In particular,

$$
\sup _{n \geqslant 0} Q\left(\left(Z_{n}^{x}\right)^{2}\right)=P \otimes P\left(\mathrm{e}^{\lambda_{2}(\beta) N_{1, \infty}}\right) .
$$

We have the following equivalence

$$
P \otimes P\left(\mathrm{e}^{\lambda_{2}(\beta) N_{1, \infty}}\right)<\infty \quad \Longleftrightarrow \quad \lambda_{2}(\beta)<\ln \left(\frac{1}{\pi_{d}}\right)
$$

where $\pi_{d} \stackrel{\text { def. }}{=} P\left(\exists n \geqslant 1, \omega_{n}=0\right)<1$. Thus, we have the following equivalence:

$$
\sup _{n \geqslant 0} Q\left(\left(Z_{n}^{x}\right)^{2}\right)<\infty \quad \Longleftrightarrow \quad \lambda_{2}(\beta)<\ln \left(\frac{1}{\pi_{d}}\right) .
$$

A series of articles $[7,1,11]$ lead to the following central limit theorem:

Theorem 2.2 ((Central limit theorem)). Suppose that the normalized partition function is bounded in $L^{2}$ :

$$
\lambda_{2}(\beta)<\ln \left(\frac{1}{\pi_{d}}\right) .
$$

Then, for all $f \in C\left(\mathbb{R}^{d}\right)$ with at most polynomial growth at infinity,

$$
\mu_{n}^{x}\left(f\left(\frac{\omega_{n}}{\sqrt{n}}\right)\right) \underset{n \rightarrow \infty}{\longrightarrow} \frac{1}{(2 \pi)^{d / 2}} \int_{\mathbb{R}^{d}} f\left(\frac{x}{\sqrt{d}}\right) \mathrm{e}^{-|x|^{2} / 2} \mathrm{~d} x, \quad \text { Q-a.s. }
$$


A step further is to try and prove a local limit theorem: one wants to obtain an expansion of the density $P^{x}\left(\mathrm{e}^{\beta H_{n}(\omega)-n \lambda(\beta)} 1_{\omega_{n}=y}\right)$. As mentioned in the introduction, this has been done in [10] by Sinai. In this paper, we will give a different proof of the local limit theorem which can be adapted to prove a continuous analogue in the Brownian setting.

Let us introduce a few notations that we will use in the rest of this paper. We define for $k \leqslant n$

$$
e_{k, n} \stackrel{\text { def. }}{=} \exp \left(\left(\sum_{j=k}^{n} \beta \eta\left(j, \omega_{j}\right)\right)-(n-k+1) \lambda(\beta)\right)
$$

and the time reversed analogue

$$
\overleftarrow{e}_{k, n} \stackrel{\text { def. }}{=} \exp \left(\left(\sum_{j=0}^{n-k} \beta \eta\left(n-j, \omega_{j}\right)\right)-(n-k+1) \lambda(\beta)\right)
$$

We can now recall Sinai's local limit theorem in a suitable form:

Theorem 2.3 ([10]). Let $d \geqslant 3, A>0$ and $\beta$ be such that $\lambda_{2}(\beta)<\ln \left(1 / \pi_{d}\right)$. Then, if $\left(l_{n}\right)_{n} \geqslant 0$ is a sequence of integers that tend to infinity such that $l_{n}=o\left(n^{a}\right)$ with $a<\frac{1}{2}$,

$$
P^{x}\left(e_{1, n} \mid \omega_{n}=y\right)=P^{x}\left(e_{1, l_{n}}\right) P^{y}\left(\overleftarrow{e}_{n-l_{n}, n}\right)+\delta_{n}^{x, y}
$$

with

$$
\sup _{|y-x| \leqslant A \sqrt{n}} Q\left(\left|\delta_{n}^{x, y}\right|^{2}\right) \underset{n \rightarrow \infty}{\longrightarrow} 0 .
$$

This leads to the following formulation that can be found in Sinai's article:

$$
P^{x}\left(e_{1, n} \mid \omega_{n}=y\right)=Z_{\infty}^{x} P^{y}\left(\overleftarrow{e}_{1, n}\right)+\bar{\delta}_{n}^{x, y}
$$

with

$$
\sup _{|y-x| \leqslant A \sqrt{n}} Q\left(\left|\bar{\delta}_{n}^{x, y}\right|\right) \underset{n \rightarrow \infty}{\longrightarrow} 0 .
$$

Remark 2.4. Intuitively, the local limit theorem asserts that, conditionally to the event $\left(\omega_{n}=y\right)$, the polymer only "feels" the environment at times $k$ small where it stays near $x$ and at times $k$ close to $n$ where it stays near $y$. In between, the polymer behaves like a conditioned simple random walk.

Remark 2.5. Theorem 2.3 leads to a weak form of Theorem 2.2: for all $f \in C\left(\mathbb{R}^{d}\right)$ with compact support,

$$
\mu_{n}^{x}\left(f\left(\frac{\omega_{n}}{\sqrt{n}}\right)\right) \underset{n \rightarrow \infty}{\stackrel{Q-\text { Probab. }}{\longrightarrow}} \frac{1}{(2 \pi)^{d / 2}} \int_{\mathbb{R}^{d}} f\left(\frac{x}{\sqrt{d}}\right) \mathrm{e}^{-|x|^{2} / 2} \mathrm{~d} x .
$$

This derivation can be found in [10].

Remark 2.6. At a heuristic level, we argue that the local limit theorem is a natural definition for the polymer to be diffusive (more natural than the central limit theorem itself). Roughly, the local limit theorem implies

$$
I_{n} \stackrel{\text { def. }}{=} \sum_{x \in \mathbb{Z}^{d}} \mu_{n}\left(\omega_{n}=x\right)^{2} \approx \sum_{x \in \mathbb{Z}^{d}}\left(P^{x}\left(\overleftarrow{e}_{1, n}\right)\right)^{2} q^{(n)}(x)^{2} \approx Q\left(Z_{n}^{2}\right) \times \sum_{x \in \mathbb{Z}^{d}} q^{(n)}(x)^{2} \approx \frac{C}{n^{d / 2}}
$$

With other respects, recall (e.g. [4]) that for $d=1,2$ and $\beta \neq 0$ or $d \geqslant 3$ and $\beta$ large,

$$
\exists \delta>0, \quad \varlimsup_{n \rightarrow \infty} I_{n} \geqslant \delta \quad Q \text {-a.s. }
$$

(at least if $\eta$ is unbounded in the second case). Therefore, it is natural to call these two cases "non-diffusive" as mentioned in the introduction. 


\subsection{The Brownian model}

This section is the continuous analogue of the previous one. We work on the product space $\left(\Omega^{2}, \mathcal{F}^{\otimes 2},\left(P^{x} \otimes\right.\right.$ $\left.P^{y}\right)_{x, y \in \mathbb{R}^{d}}$ ) and thus consider another $d$-dimensional Brownian motion $\left(\widetilde{\omega}_{t}\right)_{t \in \mathbb{R}_{+}}$independent of the first one $\left(\omega_{t}\right)_{t \in \mathbb{R}_{+}}$ under the same environment.

Let $\lambda_{2}(\beta) \stackrel{\text { def. }}{=} \lambda(2 \beta)-2 \lambda(\beta)$ where we recall that $\lambda(\beta)=\mathrm{e}^{\beta}-1$. Let $N_{s, t}=N_{s, t}(\omega, \widetilde{\omega})$ be the volume of the overlap in time $[s, t]$ of unit "tubes" around $\omega$ and $\widetilde{\omega}$ :

$$
N_{s, t} \stackrel{\text { def. }}{=} \int_{s}^{t}\left|U\left(\omega_{u}\right) \cap U\left(\widetilde{\omega}_{u}\right)\right| \mathrm{d} u .
$$

With these notations, we can find the following proposition in [3]:

Proposition 2.7. We have the following identity:

$$
Q\left(\left(Z_{t}^{x}\right)^{2}\right)=P^{x} \otimes P^{x}\left(\mathrm{e}^{\lambda_{2}(\beta) N_{0, t}}\right)=P \otimes P\left(\mathrm{e}^{\lambda_{2}(\beta) N_{0, t}}\right) .
$$

In particular,

$$
\sup _{t \geqslant 0} Q\left(\left(Z_{t}^{x}\right)^{2}\right)=P \otimes P\left(\mathrm{e}^{\lambda_{2}(\beta) N_{0, \infty}}\right) .
$$

There exists $\lambda(d)>0$ such that:

$$
\left.\lambda^{\prime} \in\right] 0, \lambda(d)\left[\Longleftrightarrow P \otimes P\left(\mathrm{e}^{\lambda^{\prime} N_{0, \infty}}\right)<\infty .\right.
$$

In [5], Comets and Yoshida prove the following central limit theorem:

Theorem 2.8 ((Central limit theorem)). Suppose that $\beta$ is such that:

$$
\lambda_{2}(\beta)<\lambda(d) .
$$

Then, for all $f \in C\left(\mathbb{R}^{d}\right)$ with at most polynomial growth at infinity,

$$
\mu_{t}^{x}\left(f\left(\frac{\omega_{t}}{\sqrt{t}}\right)\right) \underset{t \rightarrow \infty}{\longrightarrow} \frac{1}{(2 \pi)^{d / 2}} \int_{\mathbb{R}^{d}} f(x) \mathrm{e}^{-|x|^{2} / 2} \mathrm{~d} x, \quad Q \text {-a.s. }
$$

As in the discrete setting, we define for $s \leqslant t$

$$
e_{s, t} \stackrel{\text { def. }}{=} \mathrm{e}^{\beta \eta\left(V_{s, t}\right)-\lambda(\beta)(t-s)}
$$

where $V_{s, t}$ is the unit "tube" around the graph $\left\{\left(u, \omega_{u}\right)_{s<u \leqslant t}\right\}$ :

$$
\left.\left.V_{s, t}=\{(u, x) ; u \in] s, t\right], x \in U\left(\omega_{u}\right)\right\} .
$$

We also define the time reversed analogue:

$$
\overleftarrow{e}_{s, t} \stackrel{\text { def. }}{=} \mathrm{e}^{\beta \eta\left(\overleftarrow{V}_{s, t}\right)-\lambda(\beta)(t-s)}
$$

where

$$
\left.\left.\overleftarrow{V}_{s, t}=\{(t-u, x) ; u \in] 0, t-s\right], x \in U\left(\omega_{u}\right)\right\}
$$

We can now formulate a new result: the local limit theorem for Brownian polymers.

Theorem 2.9. Let $d \geqslant 3, A>0$ and $\beta$ be such that $\lambda_{2}(\beta)<\lambda(d)$. Then, if $\left(l_{t}\right)_{t \geqslant 0}$ is a positive function that tends to infinity such that $l_{t}=o\left(t^{a}\right)$ with $a<\frac{1}{2}$,

$$
P^{x}\left(e_{0, t} \mid \omega_{t}=y\right)=P^{x}\left(e_{0, l_{t}}\right) P^{y}\left(\overleftarrow{e}_{t-l_{t}, t}\right)+\delta_{t}^{x, y}
$$


with

$$
\sup _{|y-x| \leqslant A \sqrt{t}} Q\left(\left|\delta_{t}^{x, y}\right|^{2}\right) \underset{t \rightarrow \infty}{\rightarrow} 0 .
$$

This leads to the following formulation in $L^{1}$ :

$$
P^{x}\left(e_{0, t} \mid \omega_{t}=y\right)=Z_{\infty}^{x} P^{y}\left(\overleftarrow{e}_{0, t}\right)+\bar{\delta}_{t}^{x, y}
$$

with

$$
\sup _{|y-x| \leqslant A \sqrt{t}} Q\left(\left|\bar{\delta}_{t}^{x, y}\right|\right) \underset{t \rightarrow \infty}{\rightarrow} 0 .
$$

Remark 2.10. Remarks 2.4 and 2.5 apply here too.

\section{Proofs}

Our proof of Theorem 2.3 is based on the way bridge measures of the simple random walk relate to the measure of the simple random walk. This proof can be translated in the continuous setting because Brownian bridge measures relate to the Wiener measure in a similar way. The two main relations we use are the absolute continuity result (3.4) (relation (3.10) in the Brownian setting) and the inequality (3.6) (relation (3.13) in the Brownian setting) which can be proved by using potential theory.

\subsection{Proof of Theorem 2.3}

First we state and prove a few results that we will use in the proof of Theorem 2.3. We remind the classical local limit theorem for the simple random walk (cf. [9]):

Theorem 3.1 ((Local limit theorem)). For $n \in \mathbb{N}$ and $x \in \mathbb{Z}^{d}$, we say that $n$ and $x$ have the same parity and write $n \leftrightarrow x$ if $n+\sum_{k=1}^{d} x_{k}$ is even and we define $\bar{q}^{(n)}(x)$ to be the Gaussian approximation of $q^{(n)}(x)$ :

$$
\bar{q}^{(n)}(x) \stackrel{\text { def. }}{=} 2\left(\frac{d}{2 \pi n}\right)^{d / 2} \mathrm{e}^{-d|x|^{2} /(2 n)} .
$$

With these notations, we have:

$$
\sup _{x: n \leftrightarrow x}\left|q^{(n)}(x)-\bar{q}^{(n)}(x)\right|=\mathrm{O}\left(\frac{1}{n^{d / 2+1}}\right) .
$$

In particular,

$$
\sup _{x: n \leftrightarrow x} q^{(n)}(x)=\mathrm{O}\left(\frac{1}{n^{d / 2}}\right)
$$

and if one fixes $A>0$, there exists $c>0$ such that

$$
\inf _{\substack{x: n \leftrightarrow x \\|x| \leqslant A \sqrt{n}}} q^{(n)}(x) \geqslant c \frac{1}{n^{d / 2}} .
$$

We will need the following obvious corollary of Theorem 3.1 which can be understood as an absolute continuity result:

Corollary 3.2. Let $t \in] 0,1[$ and $A>0$. There exists a constant $C(A, d)>0$ such that:

$$
\begin{gathered}
\forall f \geqslant 0 \forall n \sup _{|y-x| \leqslant A \sqrt{n}} P^{x} \otimes P^{x}\left(f\left(\left(\omega_{k}, \widetilde{\omega}_{k}\right)_{k \leqslant\lfloor n t\rfloor} \mid \omega_{n}=y, \widetilde{\omega}_{n}=y\right)\right) \\
\leqslant \frac{C(A, d)}{(1-t)^{d}} P^{x} \otimes P^{x}\left(f\left(\left(\omega_{k}, \widetilde{\omega}_{k}\right)_{k \leqslant\lfloor n t\rfloor}\right)\right) .
\end{gathered}
$$


Proof. By developing the left-hand side of the inequality:

$$
\begin{aligned}
P^{x} \otimes & P^{x}\left(f\left(\left(\omega_{k}, \widetilde{\omega}_{k}\right)_{k \leqslant\lfloor n t\rfloor}\right) \mid \omega_{n}=y, \widetilde{\omega}_{n}=y\right) \\
= & \sum_{\substack{z_{1}, \ldots, z_{\lfloor n t\rfloor} \in \mathbb{Z}^{d} \\
\tilde{z}_{1}, \ldots, \tilde{z}_{\lfloor n t\rfloor} \in \mathbb{Z}^{d}}} q^{(1)}\left(z_{1}-x\right) \cdots q^{(1)}\left(z_{\lfloor n t\rfloor}-z_{\lfloor n t\rfloor-1}\right) q^{(1)}\left(\tilde{z}_{1}-x\right) \cdots q^{(1)}\left(\tilde{z}_{\lfloor n t\rfloor}-\tilde{z}_{\lfloor n t\rfloor-1}\right) \\
& \times f\left(z_{1}, \ldots, z_{\lfloor n t\rfloor}, \tilde{z}_{1}, \ldots, \tilde{z}_{\lfloor n t\rfloor}\right) \frac{q^{(n-\lfloor n t\rfloor)}\left(y-z_{\lfloor n t\rfloor}\right)}{q^{(n)}(y-x)} \frac{q^{(n-\lfloor n t\rfloor)}\left(y-\tilde{z}_{\lfloor n t\rfloor}\right)}{q^{(n)}(y-x)} .
\end{aligned}
$$

By the local limit Theorem 3.1,

$$
\frac{q^{(n-\lfloor n t\rfloor)}(y-z\lfloor n t\rfloor)}{q^{(n)}(y-x)} \underset{(3.2),(3.3)}{\leqslant} C^{\prime}\left(\frac{n}{n-\lfloor n t\rfloor}\right)^{d / 2} \leqslant \frac{C^{\prime}}{(1-t)^{d / 2}} .
$$

Similarly,

$$
\frac{q^{(n-\lfloor n t\rfloor)}\left(y-\tilde{z}_{\lfloor n t\rfloor}\right)}{q^{(n)}(y-x)} \leqslant \frac{C^{\prime}}{(1-t)^{d / 2}} .
$$

In order to prove Theorem 2.3, we will also need to use a result that comes from discrete potential theory. For a complete overview of potential theory for discrete Markov chains, we refer to [13].

Lemma 3.3. For $d \geqslant 3$ and $v: \mathbb{Z}^{d} \times \mathbb{Z}^{d} \rightarrow \mathbb{R}_{+}$a bounded and non-negative function, define

$$
\Phi(x, y)=P^{x} \otimes P^{y}\left(\mathrm{e}^{\sum_{k=1}^{\infty} v\left(\omega_{k}, \widetilde{\omega}_{k}\right)}\right) .
$$

\section{Suppose that}

$$
\sup _{x, y \in \mathbb{Z}^{d}} \Phi(x, y)<\infty
$$

Then there exists a constant $C \in] 0, \infty[$ such that

$$
\sup _{x, y \in \mathbb{Z}^{d}} P^{x} \otimes P^{y}\left(\mathrm{e}^{\sum_{k=1}^{n} v\left(\omega_{k}, \widetilde{\omega}_{k}\right)}\left|f\left(\omega_{n}, \widetilde{\omega}_{n}\right)\right|\right) \leqslant \frac{C}{n^{d}} \sum_{x, y \in \mathbb{Z}^{d}}|f(x, y)|
$$

for all $f$ in $L^{1}\left(\mathbb{Z}^{2 d}\right)$ and $n \geqslant 1$.

Proof. We will show inequality (3.5) for $n$ even, the case $n$ odd being similar. Let $\left(\omega_{n}\right)_{n \geqslant 0}$ denote the simple random walk on $\mathbb{Z}^{d}$. By Theorem 4.18 in [13], $\left(\omega_{2 n}\right)_{n} \geqslant 0$ satisfies the d-isoperimetric inequality $\left(I S_{d}\right.$ therein) on its underlying graph. By Remark 4.11 in [13], $\left(\omega_{2 n}, \widetilde{\omega}_{2 n}\right)_{n \geqslant 0}$ satisfies the 2d-isoperimetric inequality on its underlying graph. Consider the Markov chain in $\mathbb{Z}^{d} \times \mathbb{Z}^{d}$ with kernel:

$$
K\left((x, y),\left(x^{\prime}, y^{\prime}\right)\right)=\sum_{(z, \tilde{z}) \in \mathbb{Z}^{2 d}} \frac{1}{\Phi(x, y)} \mathrm{e}^{v(z, \tilde{z})} \mathrm{e}^{v\left(x^{\prime}, y^{\prime}\right)} p((x, y),(z, \tilde{z})) p\left((z, \tilde{z}),\left(x^{\prime}, y^{\prime}\right)\right)
$$

where $p$ is the transition kernel of $\left(\omega_{n}, \widetilde{\omega}_{n}\right)_{n \geqslant 0}$. The transition kernel $K$ is reversible with invariant measure $m(x, y)=$ $(\Phi(x, y))^{2} \mathrm{e}^{v(x, y)}$. By assumption, we have

$$
0<\inf _{x, y \in \mathbb{Z}^{d}} m(x, y) \leqslant \sup _{x, y \in \mathbb{Z}^{d}} m(x, y)<\infty .
$$

By assumption, there exists $c, C>0$ such that for all $(x, y),\left(x^{\prime}, y^{\prime}\right)$ in $\mathbb{Z}^{d}$

$$
c p^{(2)}\left((x, y),\left(x^{\prime}, y^{\prime}\right)\right) \leqslant K\left((x, y),\left(x^{\prime}, y^{\prime}\right)\right) \leqslant C p^{(2)}\left((x, y),\left(x^{\prime}, y^{\prime}\right)\right)
$$

where $p^{(2)}$ is the transition kernel of $\left(\omega_{2 n}, \widetilde{\omega}_{2 n}\right)_{n \geqslant 0}$. Therefore $K$ satisfies the 2d-isoperimetric inequality on its underlying graph. By Corollary 14.5 in [13],

$$
\sup _{x, y \in \mathbb{Z}^{d}} \frac{1}{\Phi(x, y)} P^{x} \otimes P^{y}\left(\mathrm{e}^{\sum_{k=1}^{2 n} v\left(\omega_{k}, \widetilde{\omega}_{k}\right)}\left|f\left(\omega_{2 n}, \widetilde{\omega}_{2 n}\right)\right| \Phi\left(\omega_{2 n}, \widetilde{\omega}_{2 n}\right)\right) \leqslant C \frac{1}{n^{d}} \sum_{x, y \in \mathbb{Z}^{d}}|f(x, y)|
$$


for all $f$ in $L^{1}\left(\mathbb{Z}^{2 d}\right)$ and $n \geqslant 1$. The inequality (3.5) follows by using the boundedness of $v$ and the assumption on $\Phi$.

We can now state the following useful corollary of Lemma 3.3:

Corollary 3.4. Let $A>0$ and $x \in \mathbb{Z}^{d}$. Under the assumptions of Lemma 3.3, there exists $\left.C \in\right] 0, \infty[$ such that:

$$
\forall n \quad \sup _{|y-x| \leqslant A \sqrt{n}} P^{x} \otimes P^{x}\left(\mathrm{e}^{\sum_{k=1}^{n} v\left(\omega_{k}, \widetilde{\omega}_{k}\right)} \mid \omega_{n}=y, \widetilde{\omega}_{n}=y\right) \leqslant C .
$$

Proof. Let $y \in \mathbb{Z}^{d}$ be such that $|y-x| \leqslant A \sqrt{n}$. By applying inequality (3.5) with $f=1_{\{y, y\}}$, we get:

$$
P^{x} \otimes P^{x}\left(\mathrm{e}^{\sum_{k=1}^{n} v\left(\omega_{k}, \widetilde{\omega}_{k}\right)} \mid \omega_{n}=y, \widetilde{\omega}_{n}=y\right) \leqslant \frac{C}{n^{d} P^{x} \otimes P^{x}\left(\omega_{n}=y, \widetilde{\omega}_{n}=y\right)} \leqslant C_{(3.3)}^{\prime} .
$$

We can now prove Theorem 2.3.

Proof of Theorem 2.3. Let $l_{n}$ be a sequence tending to infinity and such that $\forall n l_{n} \leqslant n / 2$. First, we compare in $L^{2}$ the quantity $P^{x}\left(e_{1, n} \mid \omega_{n}=y\right)$ with $P^{x}\left(e_{1, l_{n}} e_{n-l_{n}, n} \mid \omega_{n}=y\right)$. Therefore we compute:

$$
\begin{aligned}
& Q\left(P^{x}\left(e_{1, n}-e_{1, l_{n}} e_{n-l_{n}, n} \mid \omega_{n}=y\right)\right)^{2}=P^{x} \otimes P^{x}\left(\mathrm{e}^{\lambda_{2}(\beta) N_{1, n}}-\mathrm{e}^{\lambda_{2}(\beta) N_{1, l_{n}}} \mathrm{e}^{\lambda_{2}(\beta) N_{n-l_{n}, n}} \mid \omega_{n}=y, \widetilde{\omega}_{n}=y\right) \\
& \quad \leqslant P^{x} \otimes P^{x}\left(\mathrm{e}^{\lambda_{2}(\beta) N_{1, l_{n}}} \mathrm{e}^{\lambda_{2}(\beta) N_{n-l_{n}, n}} \Delta_{n} \mid \omega_{n}=y, \widetilde{\omega}_{n}=y\right)
\end{aligned}
$$

with

$$
\Delta_{n}=\mathrm{e}^{\lambda_{2}(\beta) N_{l_{n}, n-l_{n}}}-1 .
$$

Let $\delta>0$ be such that $(1+\delta) \lambda_{2}(\beta)<\ln \left(1 / \pi_{d}\right)$. We remind that this implies:

$$
\begin{aligned}
\sup _{x, y \in \mathbb{Z}^{d}} P^{x} \otimes P^{y}\left(\mathrm{e}^{(1+\delta) \lambda_{2}(\beta) \sum_{k=1}^{\infty} 1_{\omega_{k}=\widetilde{\omega}_{k}}}\right) & =P^{x} \otimes P^{x}\left(\mathrm{e}^{(1+\delta) \lambda_{2}(\beta) \sum_{k=1}^{\infty} 1_{\omega_{k}}=\widetilde{\omega}_{k}}\right) \\
& =P \otimes P\left(\mathrm{e}^{(1+\delta) \lambda_{2}(\beta) N_{1, \infty}}\right)<\infty .
\end{aligned}
$$

Using inequality (3.6) with $v(x, y)=(1+\delta) \lambda_{2}(\beta) 1_{x=y}$, there exists $C>0$ such that:

$$
\sup _{n,|y-x| \leqslant A \sqrt{n}} P^{x} \otimes P^{x}\left(\mathrm{e}^{(1+\delta) \lambda_{2}(\beta) N_{1, n}} \mid \omega_{n}=y, \widetilde{\omega}_{n}=y\right) \leqslant C .
$$

Let $\epsilon, M$ be two positive numbers such that $\mathrm{e}^{\lambda_{2}(\beta) \epsilon}-1<M$. By writing

$$
1=1_{\Delta_{n}<\mathrm{e}^{\lambda_{2}(\beta) \epsilon}-1}+1_{M<\Delta_{n}}+1_{\mathrm{e}^{\lambda_{2}(\beta) \epsilon}-1 \leqslant \Delta_{n} \leqslant M},
$$

we get

$$
\begin{aligned}
P^{x} & \otimes P^{x}\left(\mathrm{e}^{\lambda_{2}(\beta)\left(N_{1, l_{n}}+N_{n-l_{n}, n}\right)} \Delta_{n} \mid \omega_{n}=y, \widetilde{\omega}_{n}=y\right) \\
& \leqslant C\left(\mathrm{e}^{\lambda_{2}(\beta) \epsilon}-1\right)+\frac{C}{M^{\delta}}+M P^{x} \otimes P^{x}\left(1_{\Delta_{n} \geqslant \mathrm{e}^{\lambda_{2}(\beta) \epsilon}-1} \mathrm{e}^{\lambda_{2}(\beta) N_{1, n}} \mid \omega_{n}=y, \widetilde{\omega}_{n}=y\right) .
\end{aligned}
$$

Let $q>1$ be such that $\frac{1}{q}+\frac{1}{1+\delta}=1$. By Holder's inequality and inequality (3.6), we get

$$
\begin{aligned}
& P^{x} \otimes P^{x}\left(1_{\Delta_{n} \geqslant \mathrm{e}^{\lambda_{2}(\beta) \epsilon}-1} \mathrm{e}^{\lambda_{2}(\beta) N_{1, n}} \mid \omega_{n}=y, \tilde{\omega}_{n}=y\right) \\
& \quad \leqslant\left(P^{x} \otimes P^{x}\left(\Delta_{n} \geqslant \mathrm{e}^{\lambda_{2}(\beta) \epsilon}-1 \mid \omega_{n}=y, \tilde{\omega}_{n}=y\right)\right)^{1 / q} C^{1 /(1+\delta)} .
\end{aligned}
$$

But, since $N_{l_{n}, n-l_{n}}$ is integer valued, we get uniformly on $|y-x| \leqslant A \sqrt{n}$ : 


$$
\begin{aligned}
& P^{x} \otimes P^{x}\left(\Delta_{n} \geqslant \mathrm{e}^{\lambda_{2}(\beta) \epsilon}-1 \mid \omega_{n}=y, \widetilde{\omega}_{n}=y\right) \\
& \quad=P^{x} \otimes P^{x}\left(N_{l_{n}, n-l_{n}} \geqslant 1 \mid \omega_{n}=y, \widetilde{\omega}_{n}=y\right) \\
& \quad \leqslant P^{x} \otimes P^{x}\left(N_{l_{n}, n / 2} \geqslant 1 \mid \omega_{n}=y, \widetilde{\omega}_{n}=y\right)+P^{x} \otimes P^{x}\left(N_{n / 2, n-l_{n}} \geqslant 1 \mid \omega_{n}=y, \widetilde{\omega}_{n}=y\right) \\
& \quad=P^{x} \otimes P^{x}\left(N_{l_{n}, n / 2} \geqslant 1 \mid \omega_{n}=y, \widetilde{\omega}_{n}=y\right)+P^{y} \otimes P^{y}\left(N_{l_{n}, n / 2} \geqslant 1 \mid \omega_{n}=x, \widetilde{\omega}_{n}=x\right) \text { (symmetry) } \\
& \quad \leqslant C^{\prime} P^{x} \otimes P^{x}\left(N_{l_{n}, n / 2} \geqslant 1\right)+C^{\prime} P^{y} \otimes P^{y}\left(N_{l_{n}, n / 2} \geqslant 1\right) \\
& \quad(3.4) \\
& \quad=2 C^{\prime} P \otimes P\left(N_{l_{n}, n / 2} \geqslant 1\right) \underset{n \rightarrow \infty}{\longrightarrow} 0 .
\end{aligned}
$$

We have used in the limit above the fact that $N_{0, \infty}<\infty, P \otimes P$-a.s. and that $l_{n} \underset{n \rightarrow \infty}{\longrightarrow} 0$. Therefore, we get

$$
\varlimsup_{n \rightarrow \infty} \sup _{|y-x| \leqslant A \sqrt{n}} P^{x} \otimes P^{x}\left(\mathrm{e}^{\lambda_{2}(\beta)\left(N_{1, l_{n}}+N_{n-l_{n}, n}\right)} \Delta_{n} \mid \omega_{n}=y, \widetilde{\omega}_{n}=y\right) \leqslant C\left(\mathrm{e}^{\lambda_{2}(\beta) \epsilon}-1\right)+\frac{C}{M^{\delta}} .
$$

We conclude that the above limit is equal to 0 by letting $\epsilon \downarrow 0$ and $M \uparrow \infty$.

From now on, we suppose that $l_{n}=o\left(n^{a}\right)$ for some $a<\frac{1}{2}$ and denote by $P^{(z, k)}($.$) the random walk measure on$ paths which start at position $z$ at time $k$. By the Markov property of the simple random walk, we get:

$$
P^{x}\left(e_{1, l_{n}} e_{n-l_{n}, n} \mid \omega_{n}=y\right)=\sum_{\left|z_{1}-x\right| \leqslant l_{n},\left|y-z_{2}\right| \leqslant l_{n}} P^{x}\left(e_{1, l_{n}} 1_{\omega_{l n}=z_{1}}\right) \frac{q^{\left(n-2 l_{n}\right)}\left(z_{2}-z_{1}\right)}{q^{(n)}(y-x)} P^{\left(z_{2}, n-l_{n}\right)}\left(e_{n-l_{n}, n} 1_{\omega_{n}=y}\right) .
$$

By symmetry of the simple random walk, we have:

$$
\sum_{\left|y-z_{2}\right| \leqslant l_{n}} P^{\left(z_{2}, n-l_{n}\right)}\left(e_{n-l_{n}, n} 1_{\omega_{n}=y}\right)=\sum_{\left|y-z_{2}\right| \leqslant l_{n}} P^{y}\left(\overleftarrow{e}_{n-l_{n}, n} 1_{\omega_{l_{n}}=z_{2}}\right)=P^{y}\left(\overleftarrow{e}_{n-l_{n}, n}\right)
$$

Therefore,

$$
\begin{aligned}
Q & \left(\left(P^{x}\left(e_{1, l_{n}} e_{n-l_{n}, n} \mid \omega_{n}=y\right)-P^{x}\left(e_{1, l_{n}}\right) P^{y}\left(\overleftarrow{e}_{n-l_{n}, n}\right)\right)^{2}\right) \\
= & \sum_{\substack{\left|z_{1}-x\right| \leqslant l_{n},\left|y-z_{2}\right| \leqslant l_{n} \\
\left|z_{1}^{\prime}-x\right| \leqslant l_{n},\left|y-z_{2}^{\prime}\right| \leqslant l_{n}}}^{z_{n}, z_{2}, x, y} \delta_{n}^{z_{1}^{\prime}, z_{2}^{\prime}, x, y} \\
& \times P^{x} \otimes P^{x}\left(\mathrm{e}^{\lambda^{2}(\beta) N_{1, l_{n}}} 1_{\omega_{l_{n}}=z_{1}} 1_{\widetilde{\omega}_{l_{n}}=z_{1}^{\prime}}\right) P^{\left(z_{2}, n-l_{n}\right)} \otimes P^{\left(z_{2}^{\prime}, n-l_{n}\right)}\left(\mathrm{e}^{\lambda^{2}(\beta) N_{n-l_{n}, n}} 1_{\omega_{n}=y} 1_{\widetilde{\omega}_{n}=y}\right)
\end{aligned}
$$

where

$$
\delta_{n}^{z, w, x, y}=\frac{q^{\left(n-2 l_{n}\right)}(w-z)}{q^{(n)}(y-x)}-1 .
$$

The idea is that, by the classical local limit theorem, we get in the previous sum the following estimate:

$$
\frac{q^{\left(n-2 l_{n}\right)}\left(z_{2}-z_{1}\right)}{q^{(n)}(y-x)} \approx \frac{\bar{q}^{\left(n-2 l_{n}\right)}\left(z_{2}-z_{1}\right)}{\bar{q}^{(n)}(y-x)} \approx 1 .
$$

Let us make this statement rigorous and obtain inequality (3.8) below. We use the notations of Theorem 3.1 and decompose $\delta_{n}^{z, w, x, y}$ into three terms:

$$
\delta_{n}^{z, w, x, y}=\delta_{1, n}^{z, w, x, y}+\delta_{2, n}^{z, w, x, y}+\delta_{3, n}^{z, w, x, y}
$$

where

$$
\begin{aligned}
\delta_{1, n}^{z, w, x, y} & =\frac{q^{\left(n-2 l_{n}\right)}(w-z)-\bar{q}^{\left(n-2 l_{n}\right)}(w-z)}{q^{(n)}(y-x)}, \quad \delta_{2, n}^{z, w, x, y}=\frac{\bar{q}^{\left(n-2 l_{n}\right)}(w-z)-\bar{q}^{(n)}(y-x)}{q^{(n)}(y-x)}, \\
\delta_{3, n}^{z, w, x, y} & =\frac{\bar{q}^{(n)}(y-x)-q^{(n)}(y-x)}{q^{(n)}(y-x)} .
\end{aligned}
$$


An application of (3.1) and (3.3) gives for $j=1,3$ :

$$
\sup _{\substack{|z-x| \leqslant l_{n},|y-w| \leqslant l_{n} \\|y-x| \leqslant A \sqrt{n}}}\left|\delta_{j, n}^{z, w, x, y}\right|=\mathrm{O}\left(\frac{1}{n}\right) \underset{n \rightarrow \infty}{\longrightarrow} 0 .
$$

An application of (3.3) gives:

$$
\begin{aligned}
\left|\delta_{2, n}^{z, w, x, y}\right| & =\left|\frac{\bar{q}^{\left(n-2 l_{n}\right)}(w-z)}{q^{(n)}(y-x)}\right|\left|1-\frac{\bar{q}^{(n)}(y-x)}{\bar{q}^{\left(n-2 l_{n}\right)}(w-z)}\right| \underset{(3.3)}{\leqslant C}\left|1-\frac{\bar{q}^{(n)}(y-x)}{\bar{q}^{\left(n-2 l_{n}\right)}(w-z)}\right| \\
& \leqslant C\left|1-\left(\frac{n-2 l_{n}}{n}\right)^{d / 2} \mathrm{e}^{\frac{d|w-z|^{2}}{2(n-2 l n)}-\frac{d|y-x|^{2}}{2 n}}\right| .
\end{aligned}
$$

It is not hard to show that:

$$
\sup _{\substack{|z-x| \leqslant l_{n},|y-w| \leqslant l_{n} \\|y-x| \leqslant A \sqrt{n}}}\left|1-\left(\frac{n-2 l_{n}}{n}\right)^{d / 2} \mathrm{e}^{\frac{d|w-z|^{2}}{2\left(n-2 l_{n}\right)}-\frac{d|y-x|^{2}}{2 n}}\right| \underset{n \rightarrow \infty}{\longrightarrow} 0
$$

so we have

$$
\sup _{\substack{|z-x| \leqslant l_{n},|y-w| \leqslant l_{n} \\|y-x| \leqslant A \sqrt{n}}}\left|\delta_{2, n}^{z, w, x, y}\right| \underset{n \rightarrow \infty}{\longrightarrow} 0 .
$$

Finally, we get:

$$
\begin{aligned}
& \sup _{|y-x| \leqslant A \sqrt{n}} Q\left(\left(P^{x}\left(e_{1, l_{n}} e_{n-l_{n}, n} \mid \omega_{n}=y\right)-P^{x}\left(e_{1, l_{n}}\right) P^{y}\left(\overleftarrow{e}_{n-l_{n}, n}\right)\right)^{2}\right) \\
& \leqslant \sup _{\substack{|z-x| \leqslant l_{n},|y-w| \leqslant l_{n} \\
|y-x| \leqslant A \sqrt{n}}}\left|\delta_{n}^{z, w, x, y}\right|^{2}\left(P \otimes P\left(\mathrm{e}^{\lambda_{2}(\beta)\left(1+N_{1, l_{n}}\right)}\right)\right)^{2} \underset{n \rightarrow \infty}{\longrightarrow} 0 .
\end{aligned}
$$

Therefore, we get the expansion (2.1). To get the expansion (2.2), observe that

$$
P^{x}\left(e_{1, l_{n}}\right) \underset{n \rightarrow \infty}{\stackrel{L^{2}(Q)}{\longrightarrow}} Z_{\infty}^{x}
$$

and, by symmetry,

$$
\sup _{y \in \mathbb{Z}^{d}} Q\left(\left(P^{y}\left(\overleftarrow{e}_{n-l_{n}, n}\right)-P^{y}\left(\overleftarrow{e}_{1, n}\right)\right)^{2}\right)=P \otimes P\left(\mathrm{e}^{\lambda_{2}(\beta)\left(1+N_{1, l_{n}}\right)}-\mathrm{e}^{\lambda_{2}(\beta)\left(1+N_{1, n-1}\right)}\right) \underset{n \rightarrow \infty}{\longrightarrow} 0
$$

\subsection{Proof of Theorem 2.9}

In order to prove Theorem 2.9, we adapt in detail the previous proof to the Brownian setting. In the discrete setting, there are three key intermediate results: the local limit Theorem 3.1, Corollaries 3.2 and 3.4. In the continuous setting, we do not need any local limit theorem since Brownian motion is already a Gaussian process. Therefore, we only require a Brownian analogue to Corollaries 3.2 and 3.4. The following construction of the Brownian bridge can be found in the appendix of [12]:

Proposition 3.5. For $x, y \in \mathbb{R}^{d}, t>0$, there exists a unique probability measure $P_{t}^{x, y}$ on $C\left([0,1], \mathbb{R}^{d}\right)$ such that for $s \in\left[0, t\left[, A \in \mathcal{F}_{s}\right.\right.$ :

$$
P_{t}^{x, y}(A)=\frac{1}{p(t, x, y)} P^{x}\left(1_{A} p\left(t-s, \omega_{s}, y\right)\right)
$$

$y \rightarrow P_{t}^{x, y}$ is a regular conditional probability of $P_{t}^{x}$ given $\omega_{t}=y$.

In the sequel, we will always work with the representation (3.9) of Brownian bridge. With this representation, we can now easily prove the Brownian analogue of Corollary 3.2: 
Corollary 3.6. Let $s \in] 0,1[$ and $A>0$. There exists a constant $C(A, d)>0$ such that

$$
\forall f \geqslant 0 \forall t>0 \quad \sup _{|y-x| \leqslant A \sqrt{t}} P^{x}\left(f\left(\left(\omega_{u}\right)_{u \leqslant s t}\right) \mid \omega_{t}=y\right) \leqslant \frac{C(A, d)}{(1-s)^{d / 2}} P^{x}\left(f\left(\left(\omega_{u}\right)_{u \leqslant s t}\right)\right) .
$$

Proof. If $|y-x| \leqslant A \sqrt{t}$ then

$$
\begin{aligned}
P^{x}\left(f\left(\left(\omega_{u}\right)_{u \leqslant s t}\right) \mid \omega_{t}=y\right) & =\frac{(2 \pi t)^{d / 2}}{(2 \pi t(1-s))^{d / 2}} \mathrm{e}^{|y-x|^{2} /(2 t)} P^{x}\left(\mathrm{e}^{-\left|y-\omega_{s t}\right|^{2} /(2 t(1-s))} f\left(\left(\omega_{u}\right)_{u \leqslant s t}\right)\right) \\
& \leqslant \frac{\mathrm{e}^{A^{2} / 2}}{(1-s)^{d / 2}} P^{x}\left(f\left(\left(\omega_{u}\right)_{u \leqslant s t}\right)\right) . \quad \square
\end{aligned}
$$

The Brownian analogue to Lemma 3.3 is a slight variation of Lemma 3.1.3 in [5].

Lemma 3.7. For $d \geqslant 3$ and $v: \mathbb{R}^{d} \rightarrow \mathbb{R}_{+}$a bounded, non-negative and compactly supported measurable function, define

$$
\Phi(x, y)=P^{x} \otimes P^{y}\left(\mathrm{e}^{\int_{0}^{\infty} v\left(\widetilde{\omega}_{s}-\omega_{s}\right) \mathrm{d} s}\right) .
$$

Suppose that

$$
\sup _{x, y \in \mathbb{R}^{2 d}} \Phi(x, y)<\infty .
$$

Then there exists a constant $C>0$ such that

$$
\sup _{x, y \in \mathbb{R}^{2 d}} P^{x} \otimes P^{y}\left(\mathrm{e}^{\int_{0}^{t} v\left(\widetilde{\omega}_{s}-\omega_{s}\right) \mathrm{d} s}\right)\left|f\left(\omega_{t}, \widetilde{\omega}_{t}\right)\right| \leqslant \frac{C}{t^{d}} \int_{\mathbb{R}^{2 d}}|f(x, y)| \mathrm{d} x \mathrm{~d} y
$$

for all $f$ in $L^{1}\left(\mathbb{R}^{2 d}\right)$ and $t>0$.

Proof. By using the same arguments as the ones in the proof of Lemma 3.1.3 in [5], all we have to prove is

$$
\forall F \in C_{c}^{\infty}\left(\mathbb{R}^{2 d}\right) \int_{\mathbb{R}^{2 d}}\left(\frac{1}{2} \nabla_{x, y} F \cdot \nabla_{x, y} \Phi-v(y-x) F(x, y) \Phi(x, y)\right) \mathrm{d} x \mathrm{~d} y=0 .
$$

Since $\left(\widetilde{\omega}_{s / 2}-\omega_{s / 2}\right)_{s \geqslant 0}$ is a Brownian motion, we have that $\Phi(x, y)=\tilde{\Phi}(y-x)$ where:

$$
\forall z \in \mathbb{R}^{d} \quad \tilde{\Phi}(z)=P^{z}\left(\mathrm{e}^{\int_{0}^{\infty} \frac{1}{2} v\left(\omega_{s}\right) \mathrm{d} s}\right) .
$$

By Eq. (3.19) in the proof Lemma 3.1.3 in [5], we have:

$$
\forall g \in C_{c}^{\infty}\left(\mathbb{R}^{d}\right) \quad \int_{\mathbb{R}^{d}}\left(\nabla_{\tilde{y}} g(\tilde{y}) \cdot \nabla_{\tilde{y}} \tilde{\Phi}-v(\tilde{y}) g(\tilde{y}) \tilde{\Phi}(\tilde{y})\right) \mathrm{d} \tilde{y}=0 .
$$

By making the change of variable $(\tilde{x}, \tilde{y})=(y+x, y-x), F(x, y)=f(\tilde{x}, \tilde{y})$ we get:

$$
\nabla_{x, y} F \cdot \nabla_{x, y} \Phi=-\left(\nabla_{\tilde{x}} f-\nabla_{\tilde{y}} f\right) \nabla_{\tilde{y}} \tilde{\Phi}+\left(\nabla_{\tilde{x}} f+\nabla_{\tilde{y}} f\right) \nabla_{\tilde{y}} \tilde{\Phi}=2 \nabla_{\tilde{y}} f \nabla_{\tilde{y}} \tilde{\Phi} .
$$

Therefore,

$$
\begin{aligned}
\forall F \in C_{c}^{\infty}\left(\mathbb{R}^{2 d}\right) \int_{\mathbb{R}^{2 d}} & \left(\frac{1}{2} \nabla_{x, y} F \cdot \nabla_{x, y} \Phi-v(y-x) F(x, y) \Phi(x, y)\right) \mathrm{d} x \mathrm{~d} y \\
& =\frac{1}{2^{d}} \int_{\mathbb{R}^{2 d}}\left(\nabla_{\tilde{y}} f(\tilde{x}, \tilde{y}) \nabla_{\tilde{y}} \tilde{\Phi}(\tilde{y})-v(\tilde{y}) f(\tilde{x}, \tilde{y}) \tilde{\Phi}(\tilde{y})\right) \mathrm{d} \tilde{x} \mathrm{~d} \tilde{y} \\
& =\frac{1}{2^{d}} \int_{\mathbb{R}^{d}}\left(\int_{\mathbb{R}^{d}}\left(\nabla_{\tilde{y}} f(\tilde{x}, \tilde{y}) \nabla_{\tilde{y}} \tilde{\Phi}(\tilde{y})-v(\tilde{y}) f(\tilde{x}, \tilde{y}) \tilde{\Phi}(\tilde{y})\right) \mathrm{d} \tilde{y}\right) \mathrm{d} \tilde{x} \underset{(3.12)}{=0 .}
\end{aligned}
$$


We can now state the following analogue to Corollary 3.4 :

Corollary 3.8. Let $A>0$ and $x, y \in \mathbb{R}^{d}$. Under the above assumptions, there exists $C>0$ such that:

$$
\forall t \quad \sup _{|y-x| \leqslant A \sqrt{t}} P^{x} \otimes P^{x}\left(\mathrm{e}^{\int_{0}^{t} v\left(\widetilde{\omega}_{s}-\omega_{s}\right) \mathrm{d} s} \mid \omega_{t}=y, \widetilde{\omega}_{t}=y\right) \leqslant C .
$$

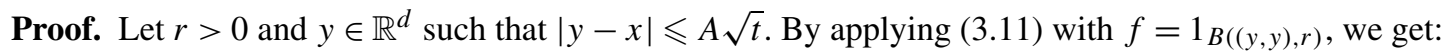

$$
P^{x} \otimes P^{x}\left(\mathrm{e}^{\int_{0}^{t} v\left(\widetilde{\omega}_{s}-\omega_{s}\right) \mathrm{d} s} 1_{B((y, y), r)}\left(\omega_{t}, \widetilde{\omega}_{t}\right)\right) \leqslant \frac{C}{t^{d}}|B((y, y), r)| .
$$

Therefore,

$$
\begin{aligned}
& P^{x} \otimes P^{x}\left(\mathrm{e}^{\int_{0}^{t} v\left(\widetilde{\omega}_{s}-\omega_{s}\right) \mathrm{d} s} 1_{B((y, y), r)}\left(\omega_{t}, \widetilde{\omega}_{t}\right)\right) \mid P^{x} \otimes P^{x}\left(\left(\omega_{t}, \widetilde{\omega}_{t}\right) \in B((y, y), r)\right) \\
& \leqslant \frac{C}{t^{d}} \frac{|B((y, y), r)|}{P^{x} \otimes P^{x}\left(\left(\omega_{t}, \widetilde{\omega}_{t}\right) \in B((y, y), r)\right)} .
\end{aligned}
$$

As $r \downarrow 0$, a classical result on Brownian bridges asserts that the left-hand side of (3.14) tends to

$$
P^{x} \otimes P^{x}\left(\mathrm{e}^{\int_{0}^{t} v\left(\widetilde{\omega}_{s}-\omega_{s}\right) \mathrm{d} s} \mid \omega_{t}=y, \widetilde{\omega}_{t}=y\right) .
$$

As $r \downarrow 0$, the right-hand side of (3.14) tends to

$$
C \frac{\mathrm{e}^{|y-x|^{2} / t}(2 \pi t)^{d}}{t^{d}} \leqslant(2 \pi)^{d} C \mathrm{e}^{A^{2}} .
$$

Proof of Theorem 2.9. The proof of Theorem 2.9 is quite similar but even simpler than the proof of Theorem 2.3 since Brownian motion is already Gaussian. We will not repeat the details but we indicate the main steps for convenience. Suppose that $\beta$ is such that

$$
\lambda_{2}(\beta)<\lambda(d) .
$$

There exists $\delta>0$ such that $(1+\delta) \lambda_{2}(\beta)<\lambda(d)$. Using inequality (3.13) applied to $v(y-x)=(1+\delta) \lambda_{2}(\beta) \mid U(y-$ $x) \cap U(0) \mid$, we get the following analogue to (3.7): there exists $C>0$ such that

$$
\sup _{|y-x| \leqslant A \sqrt{t}} P^{x} \otimes P^{x}\left(\mathrm{e}^{(1+\delta) \lambda_{2}(\beta) N_{0, t}} \mid \omega_{t}=y, \widetilde{\omega}_{t}=y\right) \leqslant C .
$$

Using inequality (3.15) and inequality (3.10), we get

$$
P^{x}\left(e_{0, t} \mid \omega_{t}=y\right) \approx P^{x}\left(e_{0, l_{t}} e_{t-l_{t}, t} \mid \omega_{t}=y\right) .
$$

Using the Markov property and the symmetry of Brownian motion, we get

$$
P^{x}\left(e_{0, l_{t}} e_{t-l_{t}, t} \mid \omega_{t}=y\right) \approx P^{x}\left(e_{0, l_{t}}\right) P^{y}\left(\overleftarrow{e}_{t-l_{t}, t}\right)
$$

\section{Acknowledgements}

I would like to thank my Ph.D. supervisor Francis Comets for his help and suggestions.

\section{References}

[1] E. Bolthausen, A note on diffusion of directed polymers in a random environment, Comm. Math. Phys. 123 (1989) 529-534.

[2] P. Carmona, Y. Hu, On the partition function of a directed polymer in a random environment, Probab. Theory Related Fields 124 (2002) 431-457.

[3] F. Comets, N. Yoshida, Brownian directed polymers in random environment, Comm. Math. Phys. 254 (2) (2004) $257-287$.

[4] F. Comets, T. Shiga, N. Yoshida, Probabilistic analysis of directed polymers in random environment, Stochastic analysis on scale interacting systems, in: Adv. Stud. Pure Math., vol. 39, Math. Soc. Japan, Tokyo, 2004, pp. 115-142.

[5] F. Comets, N. Yoshida, Some new results on Brownian directed polymers in random environment, RIMS Kokyuroku 1386 (2004) 50-66. 
[6] D.A. Huse, C.L. Henley, Pinning and roughening of domain wall in Ising systems due to random impurities, Phys. Rev. Lett. 54 (1985) 2708-2711.

[7] J.Z. Imbrie, T. Spencer, Diffusion of directed polymer in a random environment, J. Statist. Phys. 52 (3/4) (1988) 609-626.

[8] H. Krug, H. Spohn, Kinetic roughening of growing surfaces, in: C. Godrèche (Ed.), Solids Far from Equilibrium, Cambridge University Press, 1991

[9] G.F. Lawler, Intersections of Random Walks, Probab. Appl., Birkhäuser, 1991.

[10] Y. Sinai, A remark concerning random walks with random potentials, Fund. Math. 147 (1995) 173-180.

[11] R. Song, X.Y. Zhou, A remark on diffusion of directed polymers in random environment, J. Statist. Phys. 85 (1/2) (1996) $277-289$.

[12] A.S. Sznitman, Brownian Motion, Obstacles and Random Media, Springer Monogr. Math., Springer, 1998.

[13] W. Woess, Random Walks on Infinite Graphs and Groups, Cambridge University Press, 2000. 